PHYSICAL REVIEW D 95, 063522 (2017)

\title{
Unequal-time correlators for cosmology
}

\author{
T. D. Kitching ${ }^{*}$ \\ Mullard Space Science Laboratory, University College London, \\ Holmbury St. Mary, Surrey RH5 6NT, United Kingdom
}

\section{A. F. Heavens ${ }^{\dagger}$}

ICIC, Astrophysics, Imperial College, Blackett Laboratory, Prince Consort Road, London SW7 2AZ, United Kingdom

(Received 2 December 2016; published 27 March 2017)

\begin{abstract}
Measurements of the power spectrum from large-scale structure surveys have, to date, assumed an equal-time approximation, where the full cross-correlation power spectrum of the matter density field evaluated at different times (or distances) has been approximated either by the power spectrum at a fixed time or in an improved fashion, by a geometric mean $P\left(k ; r_{1}, r_{2}\right)=\left[P\left(k ; r_{1}\right) P\left(k ; r_{2}\right)\right]^{1 / 2}$. In this paper we investigate the expected impact of the geometric mean ansatz and present an application in assessing the impact on weak-gravitational-lensing cosmological parameter inference, using a perturbative unequal time correlator. As one might expect, we find that the impact of this assumption is greatest at large separations in redshift $\Delta z \gtrsim 0.3$ where the change in the amplitude of the matter power spectrum can be as much as 10 percent for $k \gtrsim 5 h \times \mathrm{Mpc}^{-1}$. However, of more concern is that the corrections for small separations, where the clustering is not close to zero, may not be negligibly small. In particular, we find that for a Euclid- or LSST-like weak lensing experiment, the assumption of equal-time correlators may result in biased predictions of the cosmic shear power spectrum, and that the impact is strongly dependent on the amplitude of the intrinsic alignment signal. To compute unequal-time correlations to sufficient accuracy will require advances in either perturbation theory to high $k$ modes or extensive use of simulations.
\end{abstract}

DOI: 10.1103/PhysRevD.95.063522

\section{INTRODUCTION}

We consider evolving scalar fields in cosmology. We define an evolving three-dimensional random field $A(\mathbf{x} ; r(t))$, where $\mathbf{x}$ labels a local three-dimensional coordinate defined at a time $t ; t$ equivalently labels a coordinate distance $r(t)$, and we may use these interchangeably. For such an evolving three-dimensional random field, we can choose a time coordinate $t$ and define a local plane-wave Fourier transform as

$$
A(\mathbf{k} ; r(t))=\int d^{3} \mathbf{x} e^{-i \mathbf{k} \cdot \mathbf{x}} A(\mathbf{x} ; r(t))
$$

where $k$ is a three-dimensional wave number, and we use the notation of [1]. Even this equation involves a subtlety; we observe this on the past light cone, so measurements are not made at a single time slice. In writing this we evolve the fields to a common time slice, and we assume in this paper that the integration volume and evolution are small enough that this can be done essentially perfectly. This then ensures that universal homogeneity is preserved in these local fields labeled with $t$.

\footnotetext{
t.kitching@ucl.ac.uk

†.heavens@imperial.ac.uk
}

The field considered could be, for instance, the density fluctuation $\delta \rho(\mathbf{x}) / \rho$ or the Newtonian potential $\Phi(\mathbf{x})$ defined at time $t_{i}$. For any two fields, each with a time label, the cross-correlation power spectrum, which is assumed to be isotropic, is defined by

$$
\begin{aligned}
& \left\langle A\left(\mathbf{k} ; r\left(t_{1}\right)\right) A^{*}\left(\mathbf{k}^{\prime} ; r\left(t_{2}\right)\right)\right\rangle \\
& \quad=(2 \pi)^{3} \delta^{3}\left(k-\mathbf{k}^{\prime}\right) P\left(k ; r\left(t_{1}\right), r\left(t_{2}\right)\right),
\end{aligned}
$$

and we have exploited the homogeneity discussed above. From here we will use the notation $r_{i}=r\left(t_{i}\right)$ for clarity. The projection of a field $A$ on the sky is defined using projection kernels $F_{A}$ such that

$$
\tilde{A}(\hat{\mathbf{n}} ; r)=\int_{0}^{r} d r_{1} F_{A}\left(r, r_{1}\right) A\left(r_{1} \hat{\mathbf{n}} ; r_{1}\right),
$$

where $r$ is a surface that labels the projection and characterizes the time when the projection is performed, and $\hat{\mathbf{n}}$ represents the direction on the sky. A simple example of $F_{A}\left(r, r_{1}\right)$ is a Gaussian centered on $r$ with a certain width. Expanding $\tilde{A}$ in terms of spherical harmonics, the angular cross-power spectrum of two different projections, $C_{\ell}^{A B}\left(r, r^{\prime}\right)$, is defined as 


$$
\begin{aligned}
C_{\ell}^{A B}\left(r, r^{\prime}\right) & \equiv\left\langle\tilde{A}_{\ell m}(r) \tilde{B}_{\ell m}^{*}\left(r^{\prime}\right)\right\rangle \\
& =\int_{0}^{r} d r_{1} \int_{0}^{r} d r_{2} F_{A}\left(r, r_{1}\right) F_{B}\left(r^{\prime}, r_{2}\right) \int \frac{d^{3} \mathbf{k}}{(2 \pi)^{3}} P\left(k ; r_{1}, r_{2}\right)(4 \pi)^{2} j_{\ell}\left(k r_{1}\right) j_{\ell}\left(k r_{2}\right) Y_{\ell m}(\hat{\mathbf{k}}) Y_{\ell m}^{*}(\hat{\mathbf{k}}) \\
& =\int_{0}^{r} d r_{1} \int_{0}^{r^{\prime}} d r_{2} F_{A}\left(r, r_{1}\right) F_{B}\left(r^{\prime}, r_{2}\right) \int \frac{2 d k k^{2}}{\pi} P\left(k ; r_{1}, r_{2}\right) j_{\ell}\left(k r_{1}\right) j j_{\ell}\left(k r_{2}\right),
\end{aligned}
$$

where $j_{\ell}$ are spherical Bessel functions of order $\ell$ and $Y_{\ell m}$ are spherical harmonics. Here we consider the case in which two fields $A$ and $B$ are different projections of the same underlying field and hence probe the same underlying power spectrum $P\left(k ; r_{1}, r_{2}\right)$ but via different projection kernels $F_{A}\left(r, r_{1}\right)$ and $F_{B}\left(r, r_{2}\right)$, respectively. This is easily generalized to different fields, in which case the crosspower spectrum is involved.

In the full expression of Eq. (4), the power spectrum $P\left(k ; r_{1}, r_{2}\right)$ is that between two different, unequal, times. We refer to $P\left(k ; r_{1}, r_{2}\right)$ as the unequal-time correlator. In cosmology, however, underlying power spectra are usually only expressed as a function of $k$ and a single time, i.e., $P(k ; r)$, encoding the Fourier correlations at a given comoving distance, but not correctly describing the correlations between comoving distances.

To overcome this issue, a mixed equal-time approximation was introduced in cosmology [2], where the geometric mean of the two equal-time power spectra has been assumed: $P\left(k ; r_{1}, r_{2}\right) \simeq\left[P\left(k ; r_{1}\right) P\left(k ; r_{2}\right)\right]^{1 / 2}$. This approximation is justified by assuming that the correlation of the underlying (matter overdensity) field is restricted to small scales in cosmology, and that over such scales the look-back time is approximately equal $\left(r_{1} \simeq r_{2}\right)$; therefore, either $P\left(k ; r_{1}\right)$ or $P\left(k ; r_{2}\right)$ could be used instead of $P\left(k ; r_{1}, r_{2}\right)$. The geometric mean approximation is then used as an algebraic convenience such that the integrals in Eq. (4) can be separated. A further justification is that the Bessel function integrals may reduce to a delta function for $r_{1} \neq r_{2}$ for large $k r$. These assumptions are tested in this paper.

\section{UNEQUAL-TIME POWER SPECTRA}

To compute the power spectrum $P\left(k ; r_{1}, r_{2}\right)$, we need to refer to the correlation between the underlying fields in Eq. (2). In the cosmological context, the underlying field of interest is often the matter overdensity $\delta(\mathbf{x}, t)=$ $[\rho(\mathbf{x}, t)-\bar{\rho}] / \bar{\rho}$, where $\rho(\mathbf{x}, t)$ is the matter density at a position $\mathbf{x}$ and comoving time $t$, and $\bar{\rho}$ is the mean matter density. This can be expanded $[3,4]$ perturbatively in terms of a growth factor $D(t)$, which is independent of scale, and a transfer function

$$
\delta(\mathbf{k}, t)=\sum_{n=1}^{\infty} D^{n}(t) f_{n}(\mathbf{k})
$$

where $\delta(\mathbf{k}, t)$ is the three-dimensional Fourier transform of $\delta(\mathbf{x}, t)$. Note that $f_{n}(\mathbf{k})$ involves products and integrals of $n$ terms of $\delta_{\mathrm{L}}(\mathbf{k}, t)$-which is the density field that can be computed from linear gravitational theory [3]. For $n>3$ such terms become complicated and numerically challenging to compute.

We can now take correlations of Eq. (5) in both the unequal-time and the equal-time cases. For the unequaltime correlation we have

$$
\begin{aligned}
P^{\mathrm{UETC}}\left(k ; r, r^{\prime}\right)= & \left\langle\delta(\mathbf{k}, t) \delta^{*}\left(\mathbf{k}, t^{\prime}\right)\right\rangle \\
= & \sum_{n=1}^{\infty} \sum_{m=1}^{\infty} D^{n}(t) D^{m}\left(t^{\prime}\right)\left\langle f_{n}(\mathbf{k}) f_{m}^{*}(\mathbf{k})\right\rangle \\
= & \sum_{n=1}^{\infty} \sum_{m=1}^{\infty} D^{n}(t) D^{m}\left(t^{\prime}\right) P_{n m}(k) \\
= & D(t) D\left(t^{\prime}\right) P_{11}(k)+D^{2}(t) D^{2}\left(t^{\prime}\right) P_{22}(k) \\
& +\left[D^{3}(t) D\left(t^{\prime}\right)+D(t) D^{3}\left(t^{\prime}\right)\right] P_{13}(k)+\cdots,
\end{aligned}
$$

where $P_{n m}(k)$ are the power spectra corresponding to the perturbatively expanded $\delta(\mathbf{k}, t)$ at order $n m$ (see e.g., [4]). Note that $D^{n}(t)$ is the linear growth factor at comoving time $t$ to the $n$th power. In the fourth line we expand this expression to include all terms up to $P_{13}(k)$.

The equal-time case can be written as

$$
\begin{aligned}
P^{\mathrm{ETC}}(k ; r(t))= & \left\langle\delta(\mathbf{k}, t) \delta^{*}(\mathbf{k}, t)\right\rangle \\
= & \sum_{n=1}^{\infty} \sum_{m=1}^{\infty} D^{n}(t) D^{m}(t)\left\langle f_{n}(\mathbf{k}) f_{m}^{*}(\mathbf{k})\right\rangle \\
= & \sum_{n=1}^{\infty} \sum_{m=1}^{\infty} D^{n}(t) D^{m}(t) P_{n m}(k) \\
= & D^{2}(t) P_{11}(k)+D^{4}(t) P_{22}(k) \\
& +2 D^{4}(t) P_{13}(k)+\cdots,
\end{aligned}
$$

where the functions $D$ and $P_{n m}$ are the same as in Eq. (6).

The geometric mean equal-time ansatz is $\left[P\left(k ; r, r^{\prime}\right)\right]^{2}=$ $P^{\mathrm{ETC}}(k ; r) P^{\mathrm{ETC}}\left(k ; r^{\prime}\right)$. By squaring Eq. (6) and multiplying Eq. (7) as required, the differences between the two cases become clear: 


$$
\begin{aligned}
{\left[P^{\mathrm{UETC}}\left(k ; r, r^{\prime}\right)\right]^{2}=} & D^{2}(t) D^{2}\left(t^{\prime}\right) P_{11}^{2}(k)+D^{4}(t) D^{4}\left(t^{\prime}\right) P_{22}^{2}(k)+\left[D^{2}(t)+D^{2}\left(t^{\prime}\right)\right] D^{2}(t) D^{2}(t) P_{13}^{2}(k) \\
& +2 D^{3}(t) D^{3}\left(t^{\prime}\right) P_{11}(k) P_{22}(k)+2 D^{2}(t) D^{2}\left(t^{\prime}\right)\left[D^{2}(t)+D^{2}\left(t^{\prime}\right)\right] P_{11}(k) P_{13}(k) \\
& +2 D^{3}(t) D^{3}\left(t^{\prime}\right)\left[D^{2}(t)+D^{2}\left(t^{\prime}\right)\right] P_{22}(k) P_{13}(k),
\end{aligned}
$$

and

$$
\begin{aligned}
P^{\mathrm{ETC}}(k ; r) P^{\mathrm{ETC}}\left(k ; r^{\prime}\right)= & D^{2}(t) D^{2}\left(t^{\prime}\right) P_{11}^{2}(k)+D^{4}(t) D^{4}\left(t^{\prime}\right) P_{22}^{2}(k)+4 D^{4}(t) D^{4}(t) P_{13}^{2}(k) \\
& +D^{2}(t) D^{2}\left(t^{\prime}\right)\left[D^{2}(t)+D^{2}\left(t^{\prime}\right)\right] P_{11}(k) P_{22}(k)+2 D^{2}(t) D^{2}\left(t^{\prime}\right)\left[D^{2}(t)+D^{2}\left(t^{\prime}\right)\right] P_{11}(k) P_{13}(k) \\
& +4 D^{4}(t) D^{4}\left(t^{\prime}\right) P_{22}(k) P_{13}(k),
\end{aligned}
$$

where each term can be compared. We see that many terms are the same but a few coincide only if $D^{2}(t)+D^{2}\left(t^{\prime}\right)=$ $2 D(t) D\left(t^{\prime}\right)$, which evidently only holds when $D(t)=$ $D\left(t^{\prime}\right)$. From here we label comoving time with redshift $z$, where the assumption of a cosmology is implicit. In Fig. 1 we show the magnitude of this approximation as a function of redshift for a concordance cosmology. In Fig. 2 we show $P^{\mathrm{UETC}}\left(k ; r, r^{\prime}\right)$ and $\left[P^{\mathrm{ETC}}(k ; r) P^{\mathrm{ETC}}\left(k ; r^{\prime}\right)\right]^{1 / 2}$, calculated using Eqs. (6) and (7) [5]. We see that the impact of the equal-time approximation is largest at large redshift separations and increases in amplitude at small scales, where $P_{22}(k)$ and $P_{13}(k)$ become dominant. For separations in redshift $\left|z-z^{\prime}\right| \gtrsim 0.3$, the change in the amplitude of the matter power spectrum can be as much as $10 \%$ for $k \gtrsim 5 \mathrm{hppc}^{-1}$. However, it is not clear yet whether the large deviations at large $\left|z-z^{\prime}\right|$ are important, since the correlation of the fields is likely to be very small here. We will return to this later.

An alternative formulation of an unequal-time correlation is to use an "eikonal" phase [7-11], where the matter overdensity perturbations can be written as [10]

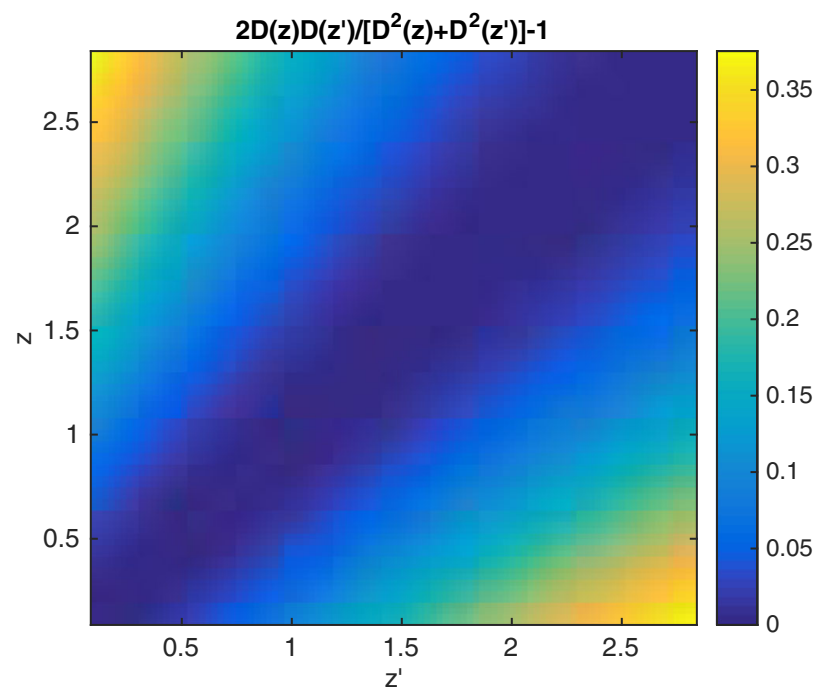

$\delta(\mathbf{k}, t) \simeq \exp \left[\int^{t} \mathrm{~d} t^{\prime} \int \frac{\mathrm{d}^{3} \mathbf{k}^{\prime}}{(2 \pi)^{3}} \frac{\mathbf{k} \cdot \mathbf{k}^{\prime}}{k^{\prime 2}} \delta_{\mathrm{L}}\left(\mathbf{k}, t^{\prime}\right)\right] \times \delta_{\mathrm{S}}(\mathbf{k}, t)$

where $\delta_{\mathrm{S}}(\mathbf{k}, r)$ represent fluctuations on short scales, and $\delta_{\mathrm{L}}(\mathbf{k}, r)$ are linear-scale perturbations; this equation assumes a growing mode only. The unequal-time power spectrum is then expressed as the equal-time version multiplied by an exponential damping term that depends on the separation in time (see for example [10], Sec. II. B), whereas the perturbative calculations in Eqs. (6) and (7) depend approximately polynomially on the eikonal phases. In this case unequal-time correlations are due to the mixing of long wavelength (or "soft") modes with shorter wavelength modes. We find that for equal times, these have no impact on the amplitude of the power spectrum, which is consistent with the conclusion we find in this section, but that for unequal times there can be significant changes [8]. Reference [12] shows how to link the eikonal phase representation to a perturbation approach similar to that

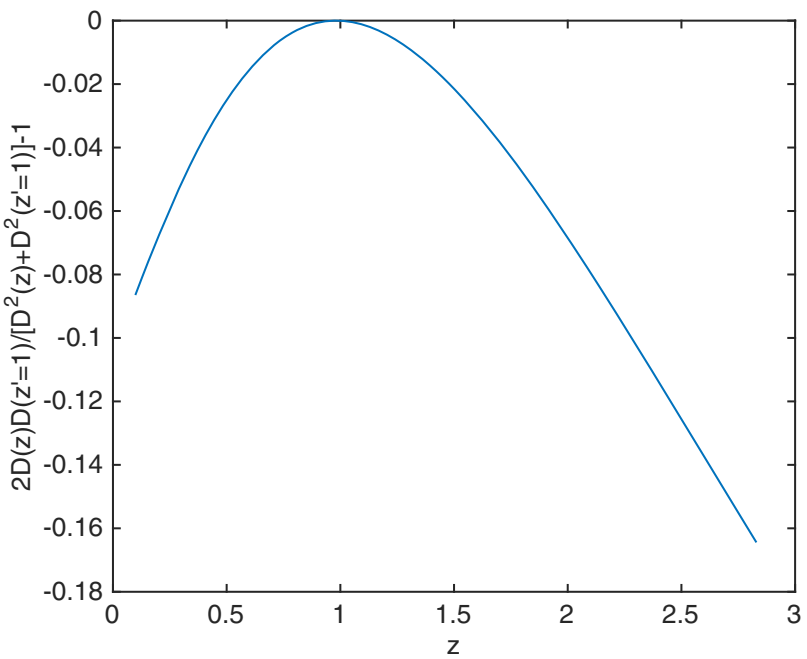

FIG. 1. Left panel: The fractional difference between $2 D(z) D\left(z^{\prime}\right)$ and $D^{2}(z)+D^{2}\left(z^{\prime}\right)$, where $D(z)$ is the linear growth factor, for a $\Lambda \mathrm{CDM}$ cosmology with parameters set at the Planck [6] maximum likelihood values. Right panel: A cross section through the left panel plot for $z^{\prime}=1$. 

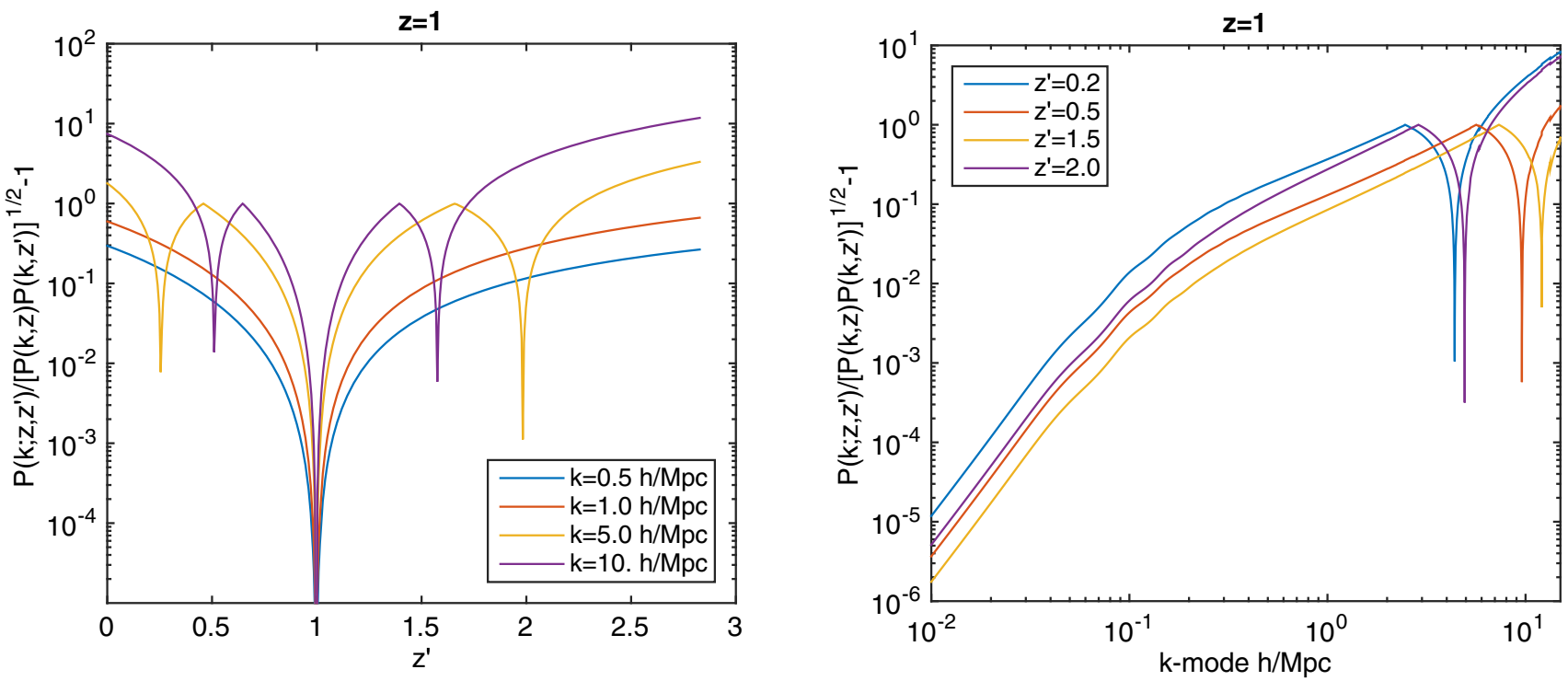

FIG. 2. The fractional difference between $P^{\mathrm{UETC}}\left(k ; r, r^{\prime}\right)$ and $\left[P^{\mathrm{ETC}}(k ; r) P^{\mathrm{ETC}}\left(k ; r^{\prime}\right)\right]^{1 / 2}$ and for a $\Lambda$ CDM cosmology with parameters set at the Planck [6] maximum likelihood values. In the left panel we set $z=1$ and show the variation as a function of $z^{\prime}$ for several $k$ modes. In the right panel we set $z=1$ and show the variation as a function of $k$ modes for several $z^{\prime}$ values.

used here. References $[10,11]$ derive power spectrum and bispectrum consistency conditions for the equal and unequal-time cases using this formalism.

It is unclear at the current time whether perturbative or eikonal phase calculations are the correct theoretical representation of cosmological power spectra. Both approaches should converge at high enough order, but given limited numerical complexity, there will be differences. For example, the eikonal phase calculations neglect some of the mode-coupling terms of the one-loop perturbative calculations (which have a $\lesssim 10 \%$ effect in the mildly nonlinear regime) but find an exponential damping for small scales and sizable time differences. One approach to address these issues is to use a matter density propagator (the sensitivity of the final to the initial conditions) (see e.g., [13]). A full and detailed comparison between these approaches is of significant interest for the case of unequal-time correlations, but it is beyond the scope of this paper.

\section{A. Bessel/Lommel effects}

Despite the fact that the amplitude of the underlying power spectrum is different in the unequal-time and equaltime cases, the integration over two Bessel functions in Eq. (4) may be expected to downweight the impact of such an effect for a projected field. Indeed, when integrating over multiple Bessel functions, an orthogonality relation holds:

$$
\int_{0}^{R} \mathrm{~d} r r^{2} j_{\ell}\left(k_{n} r\right) j_{\ell}\left(k_{m} r\right)=j_{\ell+1}\left(k_{n} R\right) \delta_{n m}^{K},
$$

where $k_{n} r$ are the arguments corresponding to zeros of the spherical Bessel functions [14,15]. However, for the general case, where nonzero parts of the spherical Bessel function are integrated over, this orthogonality relation does not hold. There have been several investigations into the regimes where this expression is applicable for cosmology $[16,17]$. In the case in which there are such integrals over $r$, such a relation can be applied in the derivation of quantities for cosmology. However, in the case of projected fields, which we investigate here, this is not appropriate because the equivalent expression that appears in Eq. (4) involves an integral over the $k$ mode. In the constant power spectrum case, and an upper $k$-mode limit of $K$, we would have expressions like

$$
\int_{0}^{K} \mathrm{~d} k k^{2} j_{\ell}\left(k r_{n}\right) j_{\ell}\left(k r_{m}\right)=j_{\ell+1}\left(K r_{n}\right) \delta_{n m}^{K},
$$

which is only applicable for a discretely sampled field in comoving distance $r_{n}$ such that $k r_{n}$ are zeros of the spherical Bessel functions; this is clearly not the case. In $[2,18,19]$ the case of $K \rightarrow \infty$ is discussed, which results in

$$
\int_{0}^{\infty} \mathrm{d} k k^{2} j_{\ell}(k r) j_{\ell}\left(k r^{\prime}\right)=\frac{\pi}{2 k^{2}} \delta^{D}\left(r-r^{\prime}\right),
$$

but this is not a practical case for cosmology; furthermore, the full expression for $K<\infty$ does not converge to the limit, as we will discuss.

To investigate the effect of the Bessel integrations, we explore the functions

$$
\begin{aligned}
C_{\ell}^{\delta \delta, \mathrm{UETC}}\left(r_{1}, r_{2}\right)= & \int \frac{2 d k k^{2}}{\pi} P^{\mathrm{UETC}}\left(k ; r_{1}, r_{2}\right) j_{\ell}\left(k r_{1}\right) j_{\ell}\left(k r_{2}\right) \\
C_{\ell}^{\delta \delta, \mathrm{ETC}}\left(r_{1}, r_{2}\right)= & \int \frac{2 d k k^{2}}{\pi}\left[P^{\mathrm{ETC}}\left(k ; r_{1}\right) P^{\mathrm{ETC}}\left(k, r_{2}\right)\right]^{1 / 2} \\
& \times j_{\ell}\left(k r_{1}\right) j_{\ell}\left(k r_{2}\right),
\end{aligned}
$$



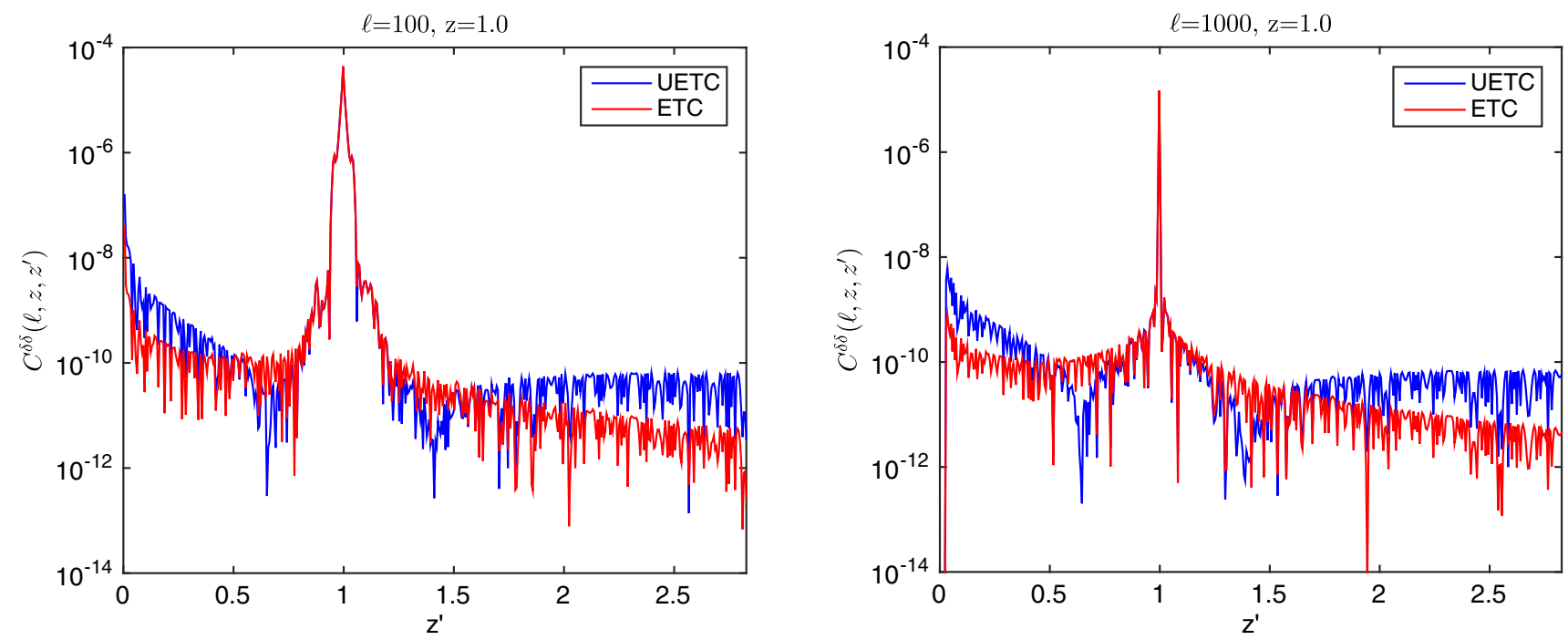

FIG. 3. The functions $C_{\ell}^{\delta \delta, \text { UETC }}\left(r_{1}, r_{2}\right)$ (blue) and $C_{\ell}^{\delta \delta \text {,ETC }}\left(r_{1}, r_{2}\right)$ (red) for several different $\ell$ modes and redshift values defined in Eq. (14). The title of each plot gives the $\ell$ mode and $z$ value used.

for the unequal-time and equal-time cases, respectively. These enter in Eq. (4) (and similarly for the equal-time case) and are then integrated over with the projection kernels $F_{A}\left(r, r_{1}\right)$ to generate the projected power spectra, $C_{\ell}^{\mathrm{AB}}\left(r, r^{\prime}\right)=\int_{0}^{r} d r_{1} \int_{0}^{r^{\prime}} d r_{2} F_{A}\left(r, r_{1}\right) F_{B}\left(r^{\prime}, r_{2}\right) C_{\ell}^{\delta \delta}\left(r_{1}, r_{2}\right)$

Equations (14) are also equal to the projected power spectra $C_{\ell}\left(r_{A}, r_{B}\right)$ when the projection kernels are delta functions e.g., $\delta^{D}\left(r-r_{1}\right)$. We assume the same $P\left(k ; r_{1}, r_{2}\right)$ as in Sec. II, where the integration is over the range $10^{-3} \leq k \leq$ $15 \mathrm{hMpc}^{-1}$ [20]. In Fig. 3 we show the functions for several different $\ell$ modes and redshift values. It can be seen that the functional form of $C_{\ell}^{\delta \delta}\left(r_{1}, r_{2}\right)$ is sharply peaked about $r_{1}=r_{2}$ but that it has a nonzero tail out to large separations $r_{1}-r_{2} \gg 1$. Again we find large differences between the unequal-time and equal-time cases, with up to a factor 100 difference for large separations in redshift. The highly oscillatory nature of the functions is a real effectnot noise in the calculation (estimated error bars from the numerical integration are smaller than the linewidth in Fig. 3). This highly oscillatory nature is a result of multiple Bessel functions in the solution moving in and out of phase as the values of $r$ change. For the case of a constant power spectrum (which is not a function of the $k$ mode), Eqs. (14) have an analytic solution given by Lommel's integrals $[14,15]$, which are also rederived in [16],

$$
\begin{aligned}
\int_{0}^{K} \mathrm{~d} k k^{2} j_{\ell}(k r) j_{\ell}\left(k r^{\prime}\right) & =\left[\frac{\pi}{2\left(r r^{\prime}\right)^{1 / 2}}\right]\left(\frac{K}{r^{2}-r^{\prime 2}}\right)\left[r J_{\ell-1}(K r) J_{\ell}\left(K r^{\prime}\right)-r_{2} J_{\ell-1}\left(K r^{\prime}\right) J_{\ell}(K r)\right] \\
\int_{0}^{K} \mathrm{~d} k k^{2} j_{\ell}(k r) j_{\ell}(k r) & =\left(\frac{\pi}{2 r}\right)\left(\frac{K^{2}}{2}\right)\left[\left(J_{\ell}(K r)\right)^{2}-J_{\ell-1}(K r) J_{\ell+1}(K r)\right],
\end{aligned}
$$

where $J$ are Bessel functions of the first kind. We note that Lommel's integrals do not converge to a delta function as $K \rightarrow \infty$ [Eq. (13)], but they become undefined. In Fig. 4 we compare the numerical calculations used in this paper with the analytic solution in Eqs. (16) and find that the difference is at least a factor of 10 smaller than the amplitude of $C^{\delta \delta}\left(\ell, z, z^{\prime}\right)$.

\section{B. Projection effects}

The final part of Eq. (4) that we consider is the effect of the projection kernels $F_{A}\left(r, r_{1}\right)$. In Fig. 3 and Eq. (14) we have already investigated the case in which $F_{A}\left(r, r_{1}\right)=$ $\delta^{D}\left(r-r_{1}\right)$. Another case that has been investigated in [1] is the case that $\left|\partial \ln F_{A}\left(r, r_{1}\right) / \partial r_{1}\right| \ll 1$, when the "Limber approximation" can be applied to Eq. (4); this is applied in the equal-time case when the limits on the $k$-mode integral are $0 \leq k \leq \infty$, and a Laplace transform can be used. We note that in the unequal-time case, the derivation of [1] is not applicable because the $r_{1}$ and $r_{2}$ integrations are not separable.

To investigate the effect of the projection kernels, we consider $B$ to be at a fixed redshift, $F_{B}\left(r, r_{2}\right)=$ $\delta^{D}\left(r-r_{2}\right)$, and use a Gaussian projection kernel for 

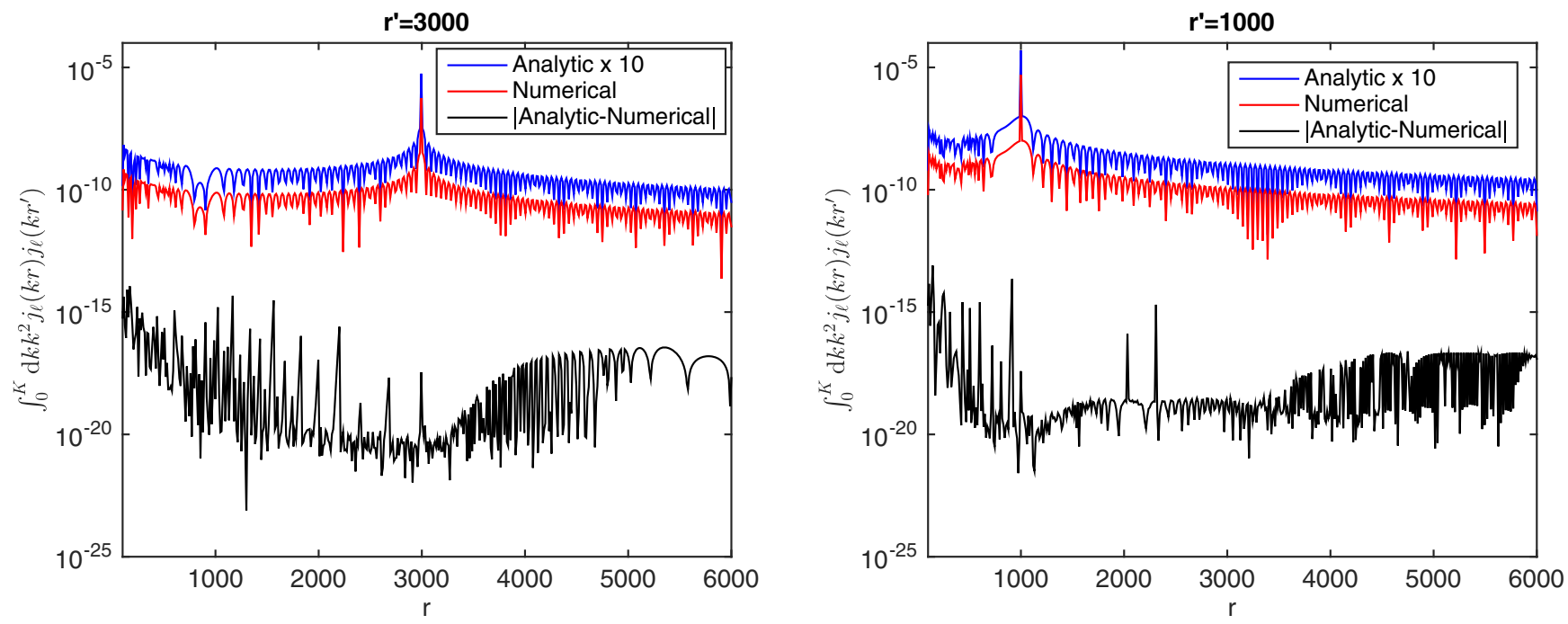

FIG. 4. Left panel: Equation (16) as a function of $r^{\prime}$ for $r=3000$. The red line is calculated using numerical integration, and the blue line is the analytic solution multiplied by ten so that the functional form can be distinguished. The black line shows the absolute difference between the two. Right panel: The same as the left except $r=1000$. Both plots are for $\ell=1000$ and $K=10 h \mathrm{Mpc}^{-1}$.

$F_{A}\left(r, r_{1}\right) \propto \exp \left[-\left(r-r_{1}\right)^{2} / 2 \sigma_{r}^{2}\right]$. We choose $r_{B}$ to be the comoving distance at $z=1$. We then vary the width of the Gaussian projection kernel $\sigma_{r}$. In Fig. 5 we show the fractional change in the projected power spectra for two different $r_{A}$ corresponding to redshifts of $z=0.5$ and 0.9 . Again the nonsmooth nature of these plots is a real feature of the oscillatory nature of the unequal-time computations.

As expected, projected cross-correlation power spectra with large redshift separations are affected more by the equal-time approximation, which can also be seen in Figs. 1 and 3. Narrow, well-separated kernels are affected the most.
Broader kernels integrate over a range of redshifts, including separations where the effect is smaller, and reducing the overall impact. We also find that high $\ell$ modes are modified more than small $\ell$ modes, which is consistent with the underlying power spectrum being affected most at high $k$ (Fig. 2). For widely separated bins in redshift, the change in the projected power spectrum can be over $10 \%$ over scales $10^{2} \lesssim \ell \lesssim 10^{4}$. Therefore, the cases in cosmology where the equal-time approximation is expected to have the greatest impact are those where widely separated tomographic redshift bins are used, with narrow projection kernels.
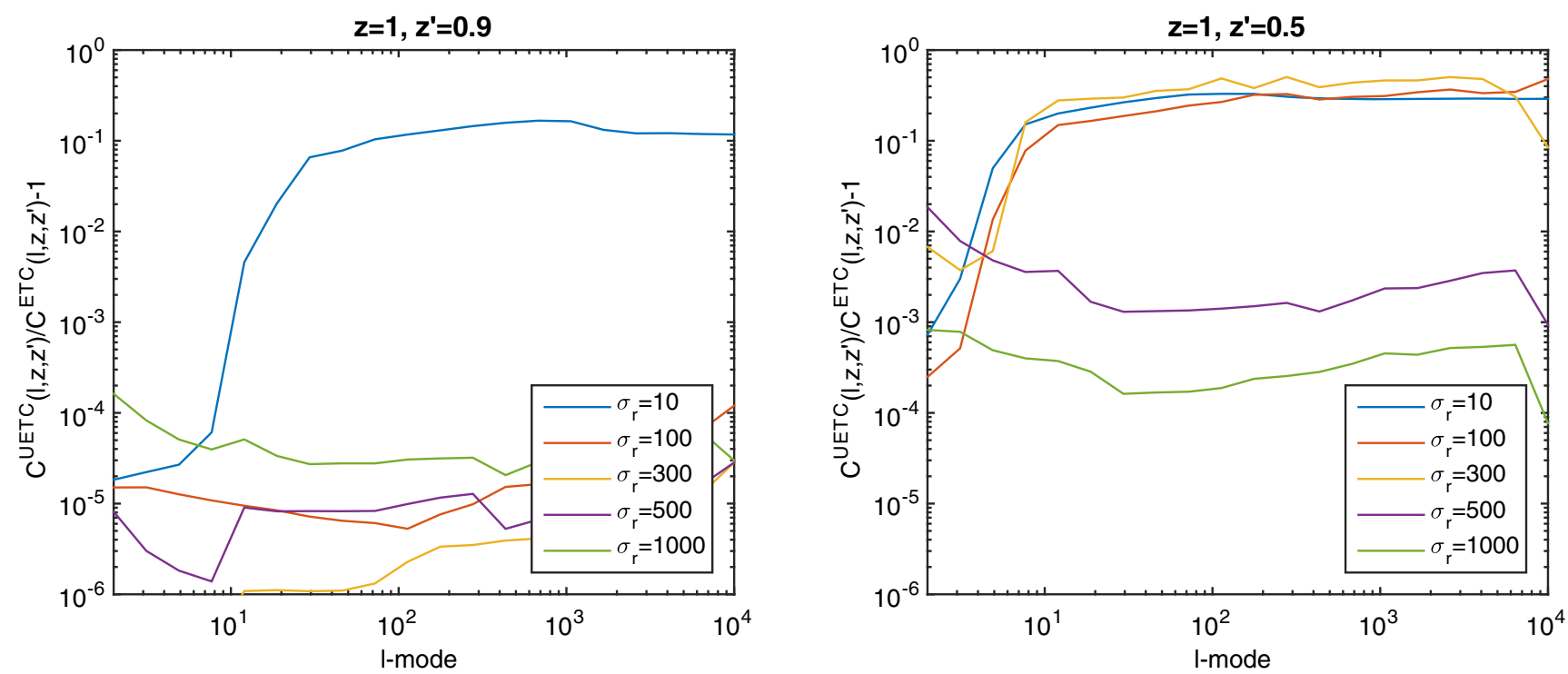

FIG. 5. Left panel: The fractional change in a projected power spectrum for a Gaussian projection kernel $F_{A}\left(r, r_{1}\right) \propto$ $\exp \left[-\left(r-r_{1}\right)^{2} / 2 \sigma_{r}^{2}\right]$, fixing $F_{B}\left(r, r_{2}\right)=\delta^{D}\left(r-r_{2}\right)$. We set $r_{B}=r(z=1)$ and $r_{A}=r\left(z^{\prime}=0.9\right)$. The lines correspond to different widths of the projection kernel. Right panel: The same as the left panel except with $r_{A}=r\left(z^{\prime}=0.5\right)$. 


\section{APPLICATION}

As shown in the previous section the difference between equal-time-approximated projected power spectra and the full unequal-time case is expected to become important for cross-correlations between widely separated redshift bins of fields that have sharply peaked projection kernels. An example of such fields in cosmology are the crosscorrelations between tomographic redshift bins of the weak lensing intrinsic alignment effect.

Weak lensing is an integrated effect, whereby the image of a distant galaxy is distorted by the gravitational lensing effect caused by matter perturbations along a line of sight and is therefore, by nature, a projected field. In weak lensing the observed ellipticity is (to first order) a sum of the following: the intrinsic (unlensed) ellipticity; the additional ellipticity caused by the gravitational lensing effect, $\gamma$, known as shear; and any noise effect, $e^{\text {obs }}=$ $e^{\text {int }}+\gamma+e^{\text {noise }}$. When taking the covariance of these quantities and constructing projected fields [as in Eq. (4)], the result is a sum of four terms:

$$
\begin{aligned}
C_{\ell}^{\mathrm{Total}}\left(r, r^{\prime}\right)= & C_{\ell}^{\mathrm{GG}}\left(r, r^{\prime}\right)+C_{\ell}^{\mathrm{GI}}\left(r, r^{\prime}\right)+C_{\ell}^{\mathrm{IG}}\left(r, r^{\prime}\right) \\
& +C_{\ell}^{\mathrm{II}}\left(r, r^{\prime}\right),
\end{aligned}
$$

where $G G$ refers to the shear-shear correlations, $G I$ to the cross-correlation between the intrinsic ellipticity and shear, and $I I$ to intrinsic-intrinsic correlations. The correlation $I G$ is expected to be zero (or at least very small), but we include it here for completeness.

The weak lensing observable is proportional to derivatives of the Newtonian potential; therefore, the inner integration over the $k$ mode in Eq. (4) is over the power spectrum of the Newtonian potential $C_{\ell}^{\Phi \Phi}\left(r_{1}, r_{2}\right)$,

$$
\begin{aligned}
& C_{\ell}^{\mathrm{GG}}\left(r, r^{\prime}\right) \\
& \quad=\int_{0}^{r} d r_{1} \int_{0}^{r^{\prime}} d r_{2} F_{G}\left(r, r_{1}\right) F_{G}\left(r^{\prime}, r_{2}\right) C_{\ell}^{\Phi \Phi}\left(r_{1}, r_{2}\right),
\end{aligned}
$$

for the $G G$ case, where the inner power spectrum is related to the matter power spectrum through Poisson's equations, which introduces a factor of $k^{-4}$ so that (up to a cosmologydependent multiplicative constant)

$$
C_{\ell}^{\Phi \Phi}\left(r_{1}, r_{2}\right)=\frac{(\ell+2) !}{(\ell-2) !} \int \frac{2 \mathrm{~d} k}{\pi k^{2}} P\left(k ; r_{1}, r_{2}\right) j_{\ell}\left(k r_{1}\right) j_{\ell}\left(k r_{2}\right),
$$

and similarly for the equal-time case, which can be compared to Eqs. (14) and (15). We include a factor of $(\ell+2) ! /(\ell-2)$ ! here, which is the result of angular derivatives relating the weak lensing observable (shear) to the projected Newtonian potential (see $[2,21]$ ).
The unlensed observed ellipticity of a galaxy can be affected by the local gravitational potential; therefore, the projection kernel in Eq. (4) is proportional to a delta function in this case. The shear effect is integrated along the line of sight, and the projection kernel is a combination of angular diameter distances that determine the geometric gravitational lensing effect. We follow the notation of $[21,22]$ and define the power spectra using Eq. (4), where the two fields are combinations of $A=\{G, I\}$ and $B=\{G, I\}$, with projection kernels given by

$$
\begin{aligned}
F_{G}\left(r, r_{1}\right) F_{G}\left(r^{\prime}, r_{2}\right) & =C_{G}(r) C_{G}\left(r^{\prime}\right) q\left(r, r_{1}\right) q\left(r^{\prime}, r_{2}\right) \\
F_{I}\left(r, r_{1}\right) F_{G}\left(r^{\prime}, r_{2}\right) & =C_{I}(r) C_{G}\left(r^{\prime}\right) p\left(r, r_{1}\right) q\left(r^{\prime}, r_{2}\right) \\
F_{G}\left(r, r_{1}\right) F_{I}\left(r^{\prime}, r_{2}\right) & =C_{G}(r) C_{I}\left(r^{\prime}\right) q\left(r, r_{1}\right) p\left(r^{\prime}, r_{2}\right) \\
F_{I}\left(r, r_{1}\right) F_{I}\left(r^{\prime}, r_{2}\right) & =C_{I}(r) C_{I}\left(r^{\prime}\right) p\left(r, r_{1}\right) p\left(r^{\prime}, r_{2}\right),
\end{aligned}
$$

where $p\left(r, r_{1}\right)$ is the comoving distance probability distribution for galaxies in a bin labeled with $r$. The shear kernel is given by

$q\left(r, r_{1}\right)=\frac{3 H_{0}^{2} \Omega_{\mathrm{M}}}{2 c^{2}} \frac{1}{a\left(r_{1}\right) r_{1}} \int \mathrm{d} r^{\prime} p\left(r, r^{\prime}\right)\left(\frac{r^{\prime}-r_{1}}{r^{\prime}}\right) w\left(r, r^{\prime}\right)$,

where $H_{0}$ is the current Hubble parameter, $\Omega_{\mathrm{M}}$ is the current dimensionless matter density, $c$ is the speed of light in vacuum, $a(r)$ is the dimensionless scale factor, $w\left(r, r^{\prime}\right)=1$ for $r^{\prime} \leq r$ and zero otherwise; here we assume a flat geometry. The functions are $C_{G}(r)=1$ and $C_{I}(r)=A_{\mathrm{IA}} c_{1} \bar{\rho}(r) / D(r)(1+z(r)) / r^{2}$, where $D(z)$ is the linear growth factor, $\bar{\rho}(z)$ is the mean matter density at redshift $z, c_{1}=5 \times 10^{-14} h^{-2} M_{\odot}^{-1} \mathrm{Mpc}^{3}$, and $A_{\mathrm{IA}}$ is a free parameter that has a fiducial value of $A_{\mathrm{IA}}=1$. This model for the intrinsic alignment power spectrum is the "linear alignment model" discussed in [23] (see [24] for a recent review).

We use a fiducial Euclid-like [25] weak lensing survey [26] (this is also similar to a LSST survey [27]), with a survey area of 15,000 square degrees, a surface number density of galaxies of $n_{0}=30$ per square arcminute, a number density distribution given by $n(z)=$ $n_{0}\left(3 z^{2} / 0.524\right) \exp \left[-(z / 0.64)^{1.5}\right]$, and a Gaussian photometric redshift distribution with standard deviation $\sigma_{z}(z)=0.03(1+z)$; the function $p\left(r, r_{1}\right)$ is given by the convolution of the $n(z)$ in each tomographic redshift bin and the photometric redshift distribution. We use five tomographic redshift bins with central redshifts given by $\{0.10,0.57,1.04,1.51,1.97\}$. We compute these power spectra for the unequal-time and equal-time cases, with the underlying power spectra given in Eqs. (6) and (7), where for the equal-time case we use the ansatz $\left[P\left(k ; r, r^{\prime}\right)\right]^{2}=P^{\mathrm{ETC}}(k ; r) P^{\mathrm{ETC}}\left(k ; r^{\prime}\right)$. 


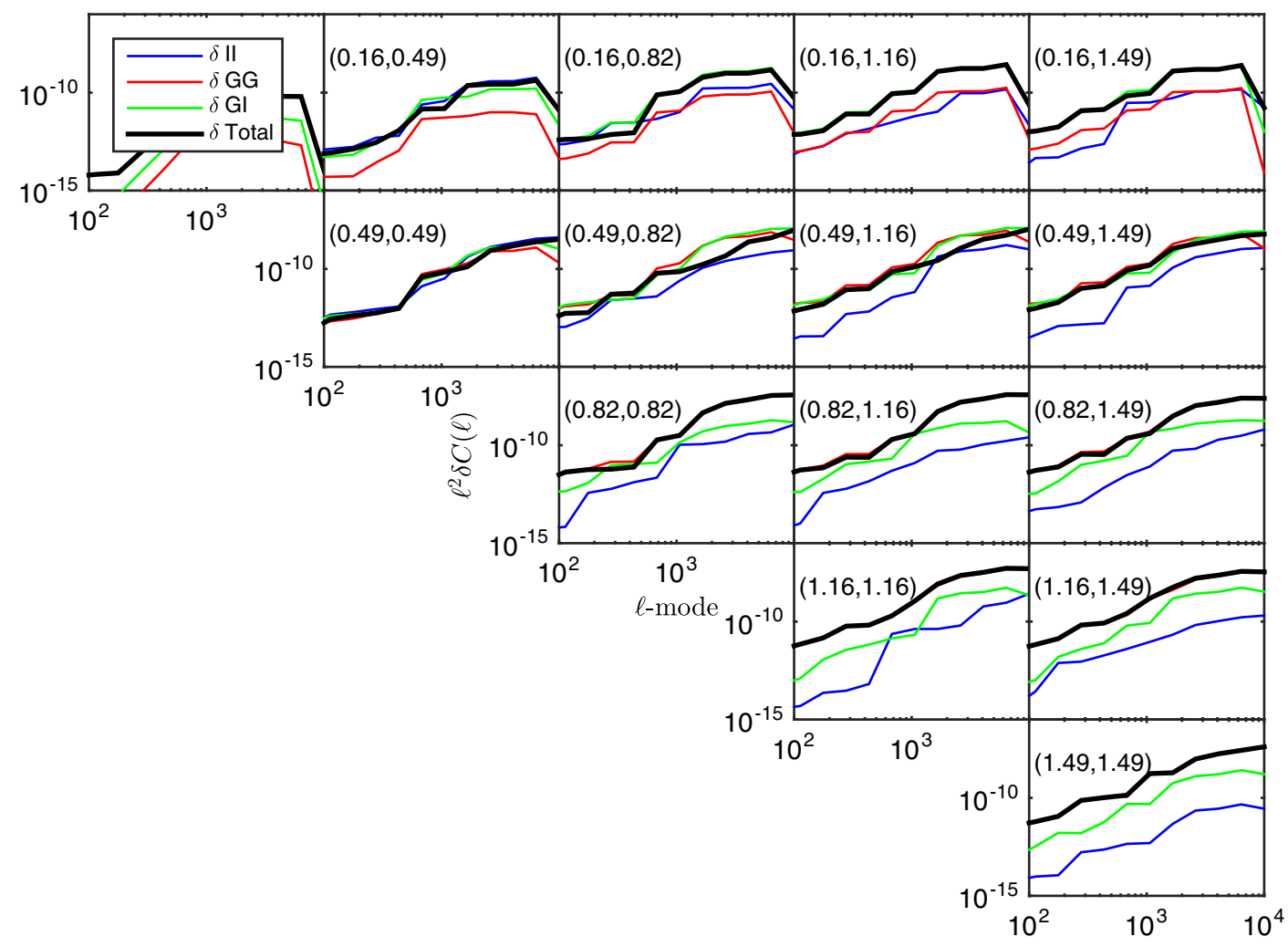

FIG. 6. The difference $\ell^{2}\left|C_{\ell}^{\mathrm{UETC}}\left(z, z^{\prime}\right)-C_{\ell}^{\mathrm{ETC}}\left(z, z^{\prime}\right)\right|$ between the unequal-time projected power spectra and the equal-time projected power spectra as a function of the $\ell$ mode for each redshift bin combination for the $G G, G I$, and $I I$ cases.

In Fig. 6 we show the difference between the projected power spectra computed in the unequal-time and equaltime cases,

$$
\delta C_{\ell}\left(z, z^{\prime}\right)=\left|C_{\ell}^{\mathrm{UETC}}\left(z, z^{\prime}\right)-C_{\ell}^{\mathrm{ETC}}\left(z, z^{\prime}\right)\right| .
$$

We find that the $G I$ term is most affected since its amplitude is relatively large and it requires a narrow kernel. The $I I$ term has a combination of two narrow kernels, but its amplitude is smaller relative to the total power spectrum. The $G G$ term is also affected but at a typically lower amplitude than the $G I$ term. If narrower redshift bins were used, then the amplitude of the difference should increase. The change in power spectrum is certainly small, but in fact the requirements on $\delta C_{\ell}\left(z, z^{\prime}\right)$ are particularly stringent for weak lensing surveys. We can assess the impact of these changes by computing the integrated effect over the differences,

$$
\langle\mathcal{A}\rangle=\frac{1}{2 \pi} \frac{1}{N_{\text {bins }}} \sum_{z \text { bins }} \int \mathrm{d} \ln \ell \ell^{2} \delta C_{\ell}\left(z, z^{\prime}\right)
$$

complementary formulations are provided for this quantity in [28-30], the sum is over all redshift bin pairs, and $N_{\text {bins }}$ is the total number of redshift bin pairs. In general, a nonzero $\langle\mathcal{A}\rangle$ will change the amplitude of the power spectrum and bias cosmological parameter inference. As discussed in
[29] the requirement on the amplitude of this quantity is $\langle\mathcal{A}\rangle \leq 10^{-7}$ for a Euclid- or LSST-like weak lensing survey to return unbiased results on the dark energy equation of state parameters; this requirement is an allowance for all systematic effects, including instrumental and algorithmic quantities. For our fiducial set we find that $\langle\mathcal{A}\rangle=7.4 \times 10^{-8}$, which would account for $\simeq 74 \%$ of the requirement [31].

Because the equal-time ansatz is expected to affect projected power spectra with narrow kernels in redshift, and because the intrinsic alignment power spectrum is such a power spectrum, we investigate the impact of changing the amplitude of the intrinsic alignment signal. In Fig. 7 we change the parameter $A_{\mathrm{IA}}$, which has a fiducial value of $A_{\mathrm{IA}}=1$, and we show how the integrated change in the power spectrum, $\langle\mathcal{A}\rangle$, varies. In the left axes we show this normalized by the required value of $10^{-7}$, and the right axes show the unnormalized value. The current range in this parameter is approximately $-1 \lesssim A_{\mathrm{IA}} \lesssim 10$ [32]. We find, as expected, that the impact of the equal-time assumption becomes more prominent as the amplitude of the intrinsic alignment signal increases.

These results serve as an example of the type of impact that the assumption of equal-time correlators may have in cosmology; however, the current precision of the perturbative approach to representing the matter power spectrum over a large range of $k$ modes, and the lack of good 


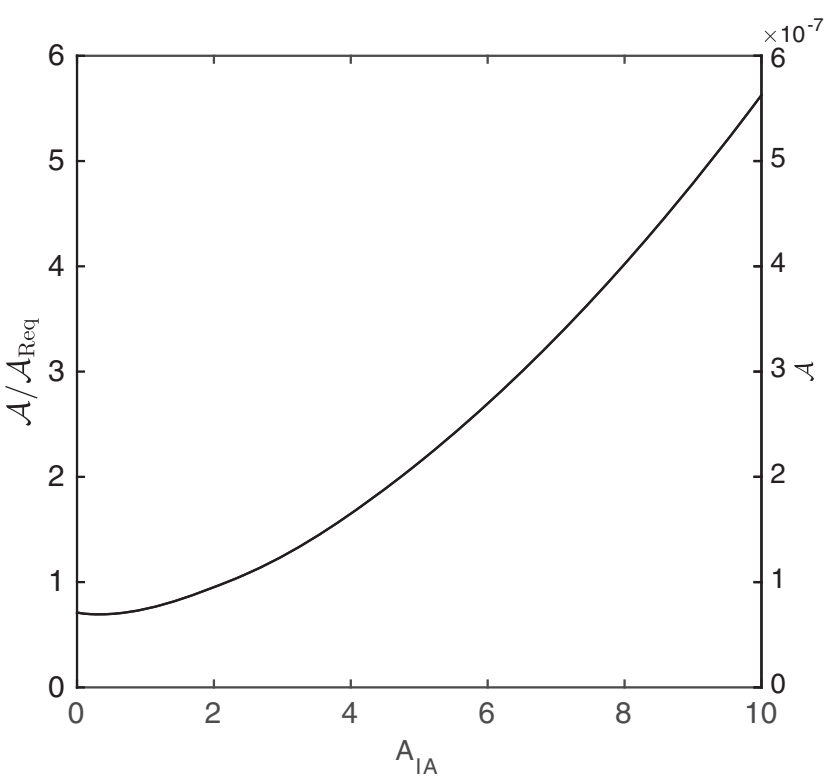

FIG. 7. The change in the integrated systematic power spectrum, Eq. (23), caused by the equal-time assumption, as a function of the intrinsic alignment amplitude $A_{\mathrm{IA}}$. In the left axes, we show this normalized by the required value of $10^{-7}$, and the right axes show the unnormalized value.

predictions for how the unequal-time power spectrum behaves as a function of cosmological parameters, shows that the precise numerical results are only indicative of the full impact, which requires further investigation.

\section{CONCLUSION}

In this paper we have presented a full projected field formalism for cosmology that includes the integration over an unequal-time correlator of the matter overdensity power spectrum $P\left(k ; r_{1}, r_{2}\right)$. This relaxes an assumption that has been made in cosmology to date where individual or mixed equal-time correlators of the matter overdensity have been used, either an ansatz $P\left(k ; r_{1}, r_{2}\right) \simeq\left[P\left(k ; r_{1}\right) P\left(k ; r_{2}\right]^{1 / 2}[2]\right.$ or alternatively simply $P\left(k ; r_{1}\right)$ or $P\left(k ; r_{2}\right)$.

We investigated the impact of the assumption of equaltime correlators by expanding both $P\left(k ; r_{1}, r_{2}\right)$ and $P\left(k ; r_{1}\right)$ perturbatively. We find that the impact of this assumption is largest at large separations in redshift $\left|z-z^{\prime}\right| \gtrsim 0.3$, where the change in the amplitude of the matter power spectrum can be as much as 5\%-10\% for $k \gtrsim 5 h \mathrm{Mpc}^{-1}$. For projected fields $C_{\ell}\left(z, z^{\prime}\right)$, we find that the impact is dependent on the width of the projection kernel. For Gaussian kernels with full width half maxima of $\sigma_{r} \lesssim 300 \mathrm{~h}^{-1} \mathrm{Mpc}$, we find a $\sim 10 \%$ effect for large redshift separations $\left|z-z^{\prime}\right| \simeq 0.5$. Therefore, observed power spectra that have narrow projection kernels and cross-correlations between widely separated redshift bins are expected to be affected by the equal-time approximation.

One application where there are narrow projected kernels and widely separated redshift bins is the computation of weak lensing two-point correlation functions and power spectra-where, in particular, the contribution from intrinsic alignments (unlensed ellipticities) has local projection kernels in comoving coordinates. We find that for a Euclidor LSST-like weak lensing experiment, the assumption of equal-time correlators could introduce biases in the measurements of cosmological parameters, and that this is strongly dependent on the amplitude of the intrinsic alignment signal. However, due to the lack of good models for how cosmological parameters affect unequal-time correlators, these results are indicative only and require further investigation.

Unequal-time correlators have been investigated previously in [33-36] in the study of cosmic strings. To determine unequal-time correlations analytically is an involved calculation, and in particular, for the matter overdensity field perturbation theory at a high $k$ mode, it would make a full analytic solution challenging. In this paper we compute the matter power spectrum using perturbation theory to third order and use $k$ modes with $k \leq 15 \mathrm{~h} \mathrm{Mpc}^{-1}$. One approach to include unequal-time correlators in cosmological statistics will be to extend this approach for arbitrary cosmological parameters and to high precision; such work has begun in studies for equal-time correlators e.g., [37,38]. As noted by [8] the eikonal phase representation of matter overdensity perturbations results in equal-time correlations being unaffected but unequal-time correlations being damped-this is consistent with the results we find here, where the equal-time case would require a lower matter power spectrum amplitude to fit the same data. Another approach will be to determine the unequal-time power spectra directly from simulations of the matter field such as $[39,40]$.

When designing statistics in cosmology, great care must be taken to investigate the impact of any assumption or approximation used. We find that the equal-time approximation can potentially have a large impact on cosmological parameter inference. Unequal-time correlators can be computed using either perturbation to high $k$ modes or from simulations, both of which require attention in order for future cosmological results to be unbiased.

\section{ACKNOWLEDGMENTS}

T. D. K. is supported by a Royal Society University Research Fellowship. We thank E. Komatsu for making his perturbatively computed power spectrum code public. 
[1] M. Loverde and N. Afshordi, Phys. Rev. D 78, 123506 (2008).

[2] P. G. Castro, A. F. Heavens, and T. D. Kitching, Phys. Rev. D 72, 023516 (2005).

[3] A. F. Heavens, S. Matarrese, and L. Verde, Mon. Not. R. Astron. Soc. 301, 797 (1998).

[4] D. Jeong and E. Komatsu, Astrophys. J. 651, 619 (2006).

[5] For these calculations we use CosmoloPy, http://roban.github io/CosmoloPy/docAPI/cosmolopy.perturbation-module.html to compute the growth factor, and the third-order perturbation code for $P_{11}, P_{22}$, and $P_{13}$ available from E. Komatsu at http://wwwmpa.mpa-garching.mpg.de/ komatsu/CRL/powerspectrum/density3pt/pkd.

[6] P. A. R. Ade, N. Aghanim, M. Arnaud, M. Ashdown, J. Aumont, C. Baccigalupi, A. J. Banday, R. B. Barreiro, J. G. Bartlett et al. (Planck Collaboration), Astron. Astrophys. 594, A13 (2016).

[7] B. Jain and E. Bertschinger, Astrophys. J. 456, 43 (1996).

[8] F. Bernardeau, N. van de Rijt, and F. Vernizzi, Phys. Rev. D 85, 063509 (2012).

[9] F. Bernardeau, N. Van de Rijt, and F. Vernizzi, Phys. Rev. D 87, 043530 (2013).

[10] I. Ben-Dayan, T. Konstandin, R. A. Porto, and L. Sagunski, J. Cosmol. Astropart. Phys. 02 (2015) 026.

[11] M. Garny, T. Konstandin, R. A. Porto, and L. Sagunski, J. Cosmol. Astropart. Phys. 11 (2015) 032.

[12] P. Creminelli, J. Gleyzes, M. Simonović, and F. Vernizzi, J. Cosmol. Astropart. Phys. 02 (2014) 051.

[13] M. Crocce and R. Scoccimarro, Phys. Rev. D 73, 063520 (2006).

[14] G. Watson, A Treatise on the Theory of Bessel Functions, Cambridge Mathematical Library (Cambridge University Press, Cambridge, England, 1995).

[15] M. Abramowitz and I. Stegun, Handbook of Mathematical Functions: With Formulas, Graphs, and Mathematical Tables, Applied Mathematics Series (Dover Publications, New York, 1964).

[16] K. B. Fisher, O. Lahav, Y. Hoffman, D. Lynden-Bell, and S. Zaroubi, Mon. Not. R. Astron. Soc. 272, 885 (1995).

[17] A. F. Heavens and A. N. Taylor, Mon. Not. R. Astron. Soc. 275, 483 (1995).

[18] N. Baddour, J. Opt. Soc. Am. A 27, 2144 (2010).

[19] B. Leistedt, A. Rassat, A. Réfrégier, and J.-L. Starck, Astron. Astrophys. 540, A60 (2012).

[20] We use the Matlab integration routine quadgk with a relative tolerance of $10^{-20}$ and an absolute tolerance of $10^{-14}$, which are sufficient for numerical convergence; see e.g., Fig. 4.

[21] T. D. Kitching, J. Alsing, A. F. Heavens, R. Jimenez, J. D. McEwen, and L. Verde, arXiv:1611.04954.
[22] T. D. Kitching, A. F. Heavens, and S. Das, Mon. Not. R. Astron. Soc. 449, 2205 (2015).

[23] C. M. Hirata and U. Seljak, Phys. Rev. D 70, 063526 (2004).

[24] B. Joachimi, M. Cacciato, T. D. Kitching, A. Leonard, R. Mandelbaum, B. M. Schäfer, C. Sifón, H. Hoekstra, A. Kiessling, D. Kirk et al., Space Sci. Rev. 193, 1 (2015).

[25] See http://euclid-ec.org.

[26] R. Laureijs, J. Amiaux, S. Arduini, J. Auguères, J. Brinchmann, R. Cole, M. Cropper, C. Dabin, L. Duvet, A. Ealet et al., arXiv:1110.3193.

[27] P. A. Abell, J. Allison, S. F. Anderson, J. R. Andrew, J. R. P. Angel, L. Armus, D. Arnett, S. J. Asztalos, T. S. Axelrod et al. (LSST Science Collaboration), arXiv:0912.0201.

[28] R. Massey, H. Hoekstra, T. Kitching, J. Rhodes, M. Cropper, J. Amiaux, D. Harvey, Y. Mellier, M. Meneghetti, L. Miller et al., Mon. Not. R. Astron. Soc. 429, 661 (2013).

[29] M. Cropper, H. Hoekstra, T. Kitching, R. Massey, J. Amiaux, L. Miller, Y. Mellier, J. Rhodes, B. Rowe, S. Pires et al., Mon. Not. R. Astron. Soc. 431, 3103 (2013).

[30] A. Amara and A. Réfrégier, Mon. Not. R. Astron. Soc. 391, 228 (2008).

[31] Using the more conservative definition for Eq. (23) in [28], which includes a normalization $N_{A}=(1 / 2 \pi)\left(1 / N_{\text {bins }}\right) \times$ $\sum_{z \text { bins }} \int \mathrm{d} \ln \ell \ell^{2}$, we find that $\langle\mathcal{A}\rangle / N_{A}=4.2 \times 10^{-13}$, which would account for $23 \%$ of the requirement.

[32] C. Heymans, E. Grocutt, A. Heavens, M. Kilbinger, T. D. Kitching, F. Simpson, J. Benjamin, T. Erben, H. Hildebrandt, H. Hoekstra et al., Mon. Not. R. Astron. Soc. 432, 2433 (2013).

[33] P. P. Avelino, E. P. S. Shellard, J. H. P. Wu, and B. Allen, Phys. Rev. Lett. 81, 2008 (1998).

[34] U.-L. Pen, U. Seljak, and N. Turok, Phys. Rev. Lett. 79, 1611 (1997).

[35] A. Albrecht, R. A. Battye, and J. Robinson, Phys. Rev. Lett. 79, 4736 (1997).

[36] A. Albrecht, R. A. Battye, and J. Robinson, Phys. Rev. D 59, 023508 (1998).

[37] G. Somogyi and R. E. Smith, Phys. Rev. D 81, 023524 (2010).

[38] D. Blas, M. Garny, T. Konstandin, and J. Lesgourgues, J. Cosmol. Astropart. Phys. 11 (2014) 039.

[39] D. Nelson, A. Pillepich, S. Genel, M. Vogelsberger, V. Springel, P. Torrey, V. Rodriguez-Gomez, D. Sijacki, G. F. Snyder, B. Griffen et al., Astron. Comput. 13, 12 (2015).

[40] J. Schaye, R. A. Crain, R. G. Bower, M. Furlong, M. Schaller, T. Theuns, C. Dalla Vecchia, C. S. Frenk, I. G. McCarthy, J. C. Helly et al., Mon. Not. R. Astron. Soc. 446, 521 (2015). 